\title{
ACA-accelerated time domain BEM for 3-D elastodynamics
}

\author{
H. Wang \\ Institute of Nuclear and New Energy Technology, \\ Tsinghua University, P. R. China
}

\begin{abstract}
This paper presents the use of the adaptive cross approximation (ACA) method for the acceleration of the classic time domain boundary element method (BEM) in 3-D elastodynamics. The main advantage of using ACA is that it is based only on the knowledge of the matrix entries; therefore no special treatment of the time domain fundamental solution is required when the coefficient matrix is approximated by using this approach. In order to discretize the boundary integral equation (BIE) we use the collocation method and eight-node discontinuous quadratic element. In the numerical examples, stability of the classic time domain BEM in terms of both the degree of discontinuity of the boundary elements and the time step size is tested, and the compression ratios of the coefficient matrices by using ACA along the time steps are studied.
\end{abstract}

Keywords: boundary element, time domain, adaptive cross approximation, elastodynamics.

\section{Introduction}

The BEM is particular suitable for the numerical treatment of elastodynamic problems in semi-infinite or infinite domains since only the boundaries need to be discretized. In addition, the radiation condition is automatically satisfied. The corresponding BIE formulations have been well established in Laplace domain [1], Fourier domain [2] and in direct time domain [3, 4]. For review of the BEM formulations in elastodynamics and applications, one can refer to the literature $[5,6]$.

Compared with the Laplace domain BEM that requires an inverse transformation, the time domain BEM provides a direct assessment of the 
investigated domains versus the time development. However, stability problems of the classic time domain BEM based on the analytic time stepping procedure have been observed and discussed [7]. In order to improve its stability, various techniques have been reported [8-11]. Besides, a Convolution Quadrature method (CQM) proposed in the literatures [12-13] has been successfully applied in the time domain BEM for dynamic problems in elastic, visco-elastic and poroelastic domains [14-17], due to its intrinsic stable feature and its ability to treat fundamental solutions in Laplace domain.

Conventionally, the BEM leads to a dense matrix. Solving such linear equation system by using standard direct or iterative solvers requires $O\left(N^{2}\right)$ memory and $O\left(N^{2} \sim N^{3}\right)$ computations, where $N$ is the number of unknowns. This is a main bottleneck for the BEM to treat large-scale problems. In order to improve its efficiency, several fast algorithms have been implemented in this field during the last several decades. Of particular interest are the fast multipole method (FMM) [18] and the adaptive cross approximation (ACA) method [19]. The FMM is an $O(N)$ algorithm based on expanding the fundamental solution into series. Therefore, FMM requires proper expansion formulation of the fundamental solution, which is problem-dependent. The ACA however, is kernel-independent and easy to be applied directly for various problems.

In the field of fast BEM in elastodynamics, one can mention the FMM-BEM in frequency domain [20-22], the FMM-BEM in time domain [23], the ACABEM in frequency domain [24] and the ACA-BEM in conjunction with CQM in time domain [25]. For the classic time domain BEM based on analytic time stepping procedure, one can note that its fundamental solutions are monotonically decreasing with the distance of $r$. This feature enables the ACA to compress the coefficient matrices at each time step. This paper presents the use of ACA to accelerate the classic time domain BEM in 3-D elastodynamics. The ACA is implemented at each time step to approximate the coefficient matrices with reasonably smaller memory and to accelerate the matrix-vector multiplications. The collocation method and the eight-node discontinuous quadratic element are used to discretize the BIE. In the numerical examples, parameters affecting stability of the classic time domain BEM are tested and determined, and efficiency of the ACA in terms of the memory requirement along the time steps is studied.

\section{Time domain BEM for 3-D elastodynamics}

The basic equations of the BEM for 3-D elastodynamics in time domain are briefly reviewed in this section. By assuming initial conditions and body forces to be zero, the time domain BIE governing elastodynamics in a 3-D homogenous domain is expressed as,

$$
\begin{aligned}
& c_{i j}(x) u_{j}(x, t)+\int_{0}^{t} \int_{S} T_{i j}^{*}(x, t ; y, \tau) u_{j}(y, \tau) d S(y) d \tau \\
& =\int_{0}^{t} \int_{S} G_{i j}^{*}(x, t ; y, \tau) t_{j}(y, \tau) d S(y) d \tau
\end{aligned}
$$


In the above, $t$ denotes time; $x$ and $y$ denote the source and field points at the boundary $S$, respectively; $u_{i}$ and $t_{i}$ are the boundary displacement and traction respectively; $c_{i j}$ is 0.5 for smooth boundaries; $G_{i j}^{*}(x, t ; y, \tau)$ and $T_{i j}^{*}(x, t ; y, \tau)$ are displacement and traction fundamental solutions respectively for the 3-D elastodynamic problems.

In order to calculate the temporal integral of Eq.(1), the time axis is discretized into $N$ steps inside which the boundary variables $u_{i}$ and $t_{i}$ are assumed to be vary linearly. Therefore, temporal integrations in Eq. (1) are done analytically.

In order to calculate the space integral of Eq. (1), we use collocation method and eight-node quadratic element. To deal with the strongly-singular integrals, the finite part integral is used. To satisfy its continuity requirements and to treat the corners in a simple manner, we adopt discontinuous elements from the literature [26] (see Fig. 1), where the positioning parameter $\lambda(0<\lambda<1)$ stands for the degree of continuity.

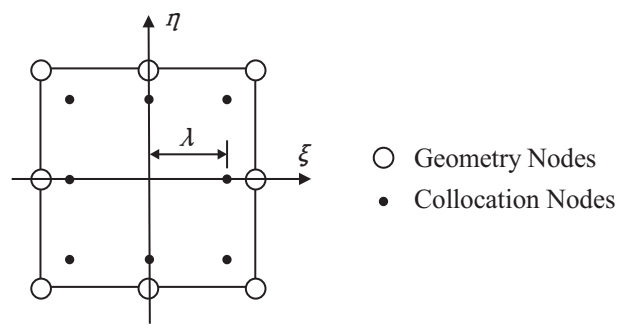

Figure 1: Discontinuous eight node quadratic element.

After collocation, time and space discretization, Eq.(1) takes a matrix form,

$$
\begin{aligned}
& {[c]\left\{u^{N}\right\}+\left[T^{1,0}\right]\left\{u^{N}\right\}=\left[G^{1,0}\right]\left\{t^{N}\right\}} \\
& +\sum_{I=1}^{N-1}\left[G^{1, I}+G^{2, I}\right]\left\{t^{N-I}\right\}-\sum_{I=1}^{N-1}\left[T^{1, I}+T^{2, I}\right]\left\{u^{N-I}\right\}+\left[G^{2, N}\right]\left\{t^{0}\right\}
\end{aligned}
$$

where $[G]$ and $[T]$ arise from the integrations of the displacement and traction fundamental solutions respectively at the $I$-th time step. Herein the first number of the superscripts of $[G]$ and $[T]$ is used to distinguish between the parts inside the integral at $I$ and $I-1$.

\section{ACA applied to time domain BEM}

This section summarizes basic idea of the ACA and procedures of the ACA applied to the time domain BEM. The ACA is an algebraic approximation method which is based only on the knowledge of the matrix entries. For a low rank matrix denoted by $[A]^{m \times n}$, approximation work can be made by using the ACA [19], 


$$
[A]^{m \times n} \approx[U]^{m \times k}[V]^{k \times n}
$$

where $k \ll m, n$. Therefore, the matrix-vector multiplication is reduced from $O(m \times n)$ to $O(k(m+n))$ in both memory and computations. There are commonly two types of ACA, namely the fully-pivoted and partially-pivoted ACA. The former approach requires all matrix entries to be calculated; the latter one requires only a small part of the matrix entries. For the vector problems such as 3-D elastodynamics, it is more convenient to calculate a set of matrix entries based on the element than to calculate a single entry. Therefore in this paper, the fully-pivoted ACA is applied.

Procedures of applying ACA to the time domain BEM are as follows

\section{Step 1:}

An adaptive tree is constructed based on the geometry information of the boundary elements. The elastic domain is then subdivided recursively into subdomains at various levels each containing a set of boundary elements.

\section{Step 2:}

At each time step, the coefficient matrices $[G]$ and $[T]$ are partitioned into submatrices at various levels. Each sub-matrix arises from the interaction of two sets of boundary elements belonging to specific subdomains at Step 1.

\section{Step 3:}

For $[G]$ and $[T]$ at each time step, identify low rank sub-matrices according to the associated geometrical and distance information of subdomains. Next for full rank sub-matrices, calculate directly; for low rank sub-matrices, perform ACA operations.

Attention should be paid when $\left[G^{1}+G^{2}\right]$ and $\left[T^{1}+T^{2}\right]$ in Eq.(4) are approximated by using ACA. In this case, the corresponding sub-matrices are added up accordingly. In the case of a low rank sub-matrix added to another, the following direct combination is used,

$$
\left[A^{1}\right]^{m \times n}+\left[A^{2}\right]^{m \times n} \approx\left[U^{1}\right]^{m \times k}\left[V^{1}\right]^{k \times n}+\left[U^{2}\right]^{m \times k}\left[\begin{array}{l}
V^{2}
\end{array}\right]^{k \times n}=\left[\begin{array}{ll}
U^{1} & U^{2}
\end{array}\right]^{m \times 2 k}\left[\begin{array}{l}
V^{1} \\
V^{2}
\end{array}\right]^{2 k \times n}
$$

\section{Step 4:}

At each time step, the boundary variables are obtained by solving the linear equation system using GMRES, in which the coefficient matrix-vector multiplications are approximated by ACA matrix-vector multiplications.

\section{Numerical examples}

A $\mathrm{C}++$ code of the ACA-BEM for 3-D elastodynamics has been developed. In the following, a 3 -D elastic rod of $2 \times 2 \times 4$ is considered. The rod is fixed at one 
end and given a pressure of $p(t)=-H(t)$ at the opposite free end, where $H(t)$ is Heaviside function. The rest boundaries are free. The Young's modulus and Poisson's ratio are 100.0 and 0.0, respectively; the density is 1.0; in GMRES the relative error for convergence is set to be $10^{-5}$; the error for stop in the fullypivoted ACA is set to be $10^{-5}$.

First, stability of the time domain BEM in terms of the degree of discontinuity of the boundary element (see $\lambda$ in Fig. 1) and the time step size is tested. The time step size is represented by defining a dimensionless parameter as,

$$
\beta=\frac{c_{1} \Delta t}{L_{e}}
$$

where $c_{1}$ is the compression wave velocity and $L_{e}$ is the representative element length. The rod is discretized into 40 discontinuous elements as shown in Fig. 2, with DOF $=960$. We choose $\lambda=1 / 2,2 / 3$ and $\beta=0.1 \sim 0.8$ (with increment of $0.1)$ respectively for the test. It is found that only a few parameter combinations result in relative good stabilities in longitudinal displacement at the free end, namely $\lambda=1 / 2, \beta=0.5,0.8$ and $\lambda=2 / 3, \beta=0.6,0.7$. In addition, $\lambda=1 / 2$ leads to much earlier instabilities than $\lambda=2 / 3$, as shown in Figs. 3 and 4 in comparison with analytic results. Therefore, the combination of $\lambda=2 / 3, \beta=0.7$ is determined for the following study.

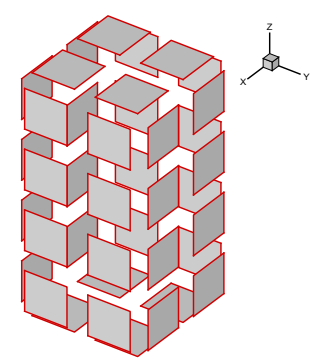

Figure 2: The rod with 40 discontinuous elements.

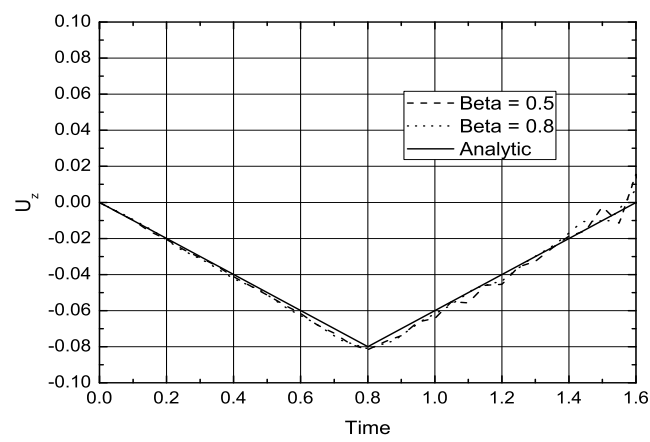

Figure 3: Displacement vs. time $(\lambda=1 / 2, \beta=0.5,0.8)$. 


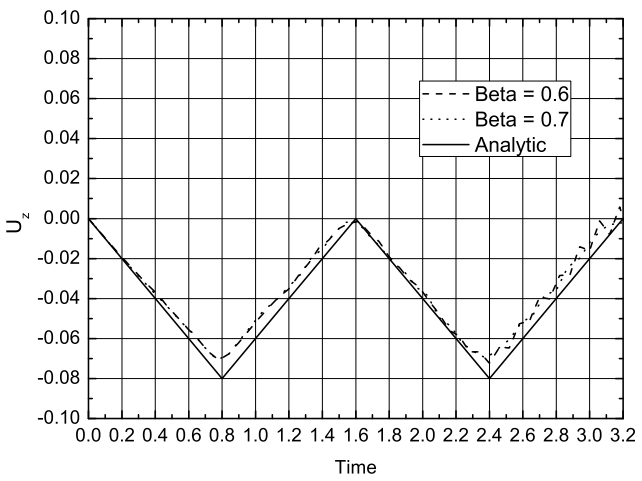

Figure 4: $\quad$ Displacement vs. time $(\lambda=2 / 3, \beta=0.6,0.7)$.

In the second test, the rod is meshed with $90(\mathrm{DOF}=2160)$ and 160 $(\mathrm{DOF}=3840)$ elements respectively. The compression ratios of $\left[G^{1}\right],\left[T^{1}\right]$ and $\left[G^{1}+G^{2}\right]$ by using ACA versus the time step are shown in Figs. 5-7. It is shown that larger DOF results in better compression ratio. It is also observed, as expected, that the trend of the compression ratio along the time axis follows that of the share of non-zero entries in the coefficient matrices.

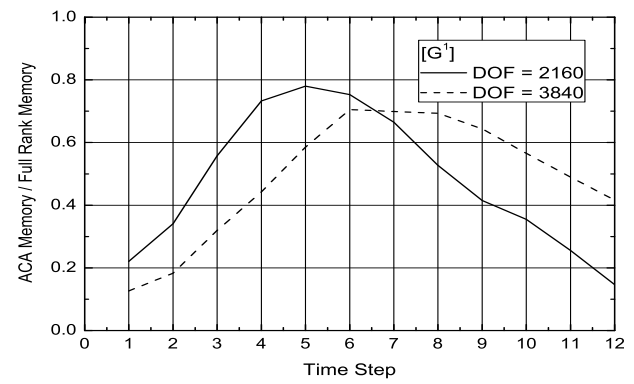

Figure 5: $\quad$ Compression ratio: $\left[G^{1}\right]$.

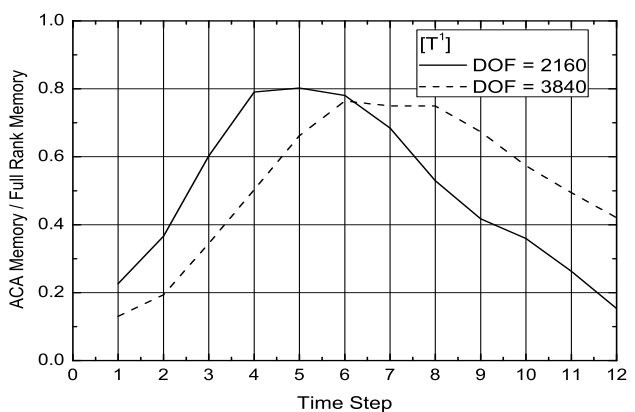

Figure 6: Compression ratio: $\left[T^{1}\right]$. 


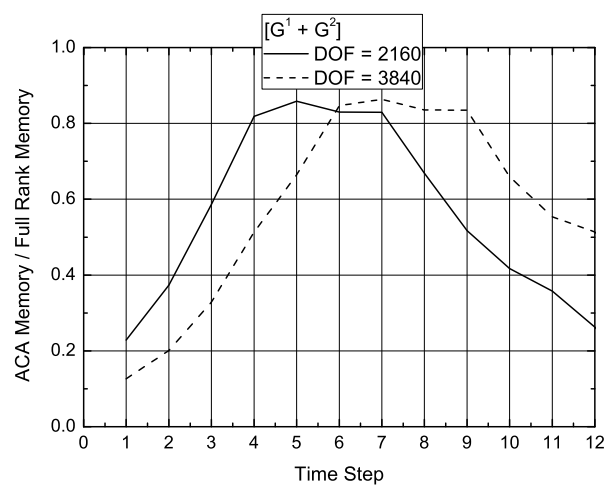

Figure 7: $\quad$ Compression ratio: $\left[G^{1}+G^{2}\right]$.

\section{Conclusions}

The use of ACA as a fast solver for the classic time domain BEM in 3-D elastodynamics was presented. The collocation method, linear time step and eight-node discontinuous quadratic element were used for discretization. The numerical tests showed the effects of some parameters on stability of the classic time domain BEM and studied compression ratios of the time domain BEM matrices by using ACA.

\section{Acknowledgement}

Financial support for the project from the National Natural Science Foundation of China, under grant No. 11072128 is gratefully acknowledged.

\section{References}

[1] Cruse, T.A. and Rizzo, F.J.A., Direct formulation and numerical solution of the general transient elastodynamic problem I. Journal of Mathematical Analysis and Applications, 22, pp. 244-259, 1968.

[2] Domínguez, J., Boundary elements in dynamics, Southampton, Boston: Computational Mechanics Publications, 1993.

[3] Mansur, W.J., A time-stepping technique to solve wave propagation problems using the boundary element method. Ph.D. Thesis, University of Southampton, 1983.

[4] Antes, H., A boundary element procedure for transient wave propagations in two-dimensional isotropic elastic media. Finite Elements in Analysis and Design, 1, pp. 313-322, 1985.

[5] Beskos, D.E., Boundary element methods in dynamic analysis. Applied Mechanics Review, 40(1), pp. 1-23, 1987. 
[6] Beskos, D.E., Boundary element methods in dynamic analysis: Part II (1986-1996). Applied Mechanics Review, 50(3), pp. 149-197, 1997.

[7] Birgisson, B., Siebrits, E. and Peirce, A.P., Elastodynamic direct boundary element methods with enhanced numerical stability properties. Int. J. Numer. Methods Engrg., 46, pp. 871-888, 1999.

[8] Peirce, A. and Siebrits, E., Stability analysis and design of time-stepping schemes for general elastodynamic boundary element models. Int. J. Numer. Methods Engrg., 40(2), pp. 319-342, 1997.

[9] Rizos, D.C. and Karabalis, D.L., An advanced direct time domain BEM formulation for general 3-D elastodynamic problems. Comput. Mech., 15, pp. 249-269, 1994.

[10] Mansur, W.J., Carrer, J.A.M. and Siqueira, E.F.N., Time discontinuous linear traction approximation in time-domain BEM scalar wave propagation. Int. J. Numer. Methods Engrg., 42(4), pp. 667-683, 1998.

[11] Yu, G., Mansur, W.J., Carrer, J.A.M. and Gong, L., Time weighting in time domain BEM. Eng. Anal. Bound. Elem., 22(3), pp. 175-181, 1998.

[12] Lubich, C., Convolution quadrature and discretized operational calculus I. Numerische Mathematik, 52, pp. 129-145, 1988.

[13] Lubich, C., Convolution quadrature and discretized operational calculus II. Numerische Mathematik, 52, pp. 413-425, 1988.

[14] Schanz, M. and Antes, H., A new visco- and elastodynamic time domain boundary element formulation. Computational Mechanics, 20(5), pp. 452 459, 1997.

[15] Gaul, L. and Schanz, M., A comparative study of three boundary element approaches to calculate the transient response of viscoelastic solids with unbounded domains. Comput. Methods Appl. Mech. Engrg., 179, pp. 111$123,1999$.

[16] Kielhorn, L. and Schanz, M., Convolution quadrature method-based symmetric Galerkin boundary element method for 3-d elastodynamics. Int. J. Numer. Meth. Engng., 76, pp. 1724-1746, 2008.

[17] Schanz, M., Application of 3D time domain boundary element formulation to wave propagation in poroelastic solids. Engineering Analysis with Boundary Elements, 25, pp. 363-376, 2001.

[18] Greengard, L. and Rokhlin, V., A fast algorithm for particle simulations. $J$. Comput. Phys., 73, pp. 325-348, 1987.

[19] Bebendorf, M. and Rjasanow, S., Adaptive low-rank approximation of collocation matrices. Computing, 70, pp. 1-24, 2003.

[20] Chen, Y.H., Chew, W.C. and Zeroug, S., Fast multiple method as an efficient solver for 2D elastic wave surface integral equations. Comp. Mech., 20, pp. 495-506, 1997.

[21] Chaillat, S., Bonnet, M. and Semblat, J.F., A multi-level fast multipole BEM for 3-D elastodynamics in the frequency domain. Computer Methods in Applied Mechanics and Engineering, 197(49-50), pp. 4233-4249, 2008.

[22] Takahashi, T., A wideband fast multipole accelerated boundary integral equation method for time-harmonic elastodynamics in two dimensions. Int. J. Numer. Meth. Engng., 91, pp. 531-551, 2012. 
[23] Takahashi, T., Nishimura, N. and Kobayashi, S., A fast BIEM for threedimensional elastodynamics in time domain. Engineering Analysis with Boundary Elements, 27, pp. 491-506, 2003.

[24] Benedetti, I. and Aliabadi, M.H., A fast hierarchical dual boundary element method for three-dimensional elastodynamic crack problems. Int. J. Numer. Methods Engng., 84, pp. 1038-1067, 2010.

[25] Messner, M. and Schanz, M., An accelerated symmetric time-domain boundary element formulation for elasticity. Engineering Analysis with Boundary Elements, 34, pp. 944-955, 2010.

Mi, Y. and Aliabadi, M.H., Dual boundary element method for threedimensional fracture mechanics analysis. Engrg. Anal. Boundary Elements, 10, pp. 161-171, 1992. 